\title{
Implementation and first operational experiences with the high voltage In-Service- Tests on the superconducting magnet system of Wendelstein 7-X
}

\author{
F. Füllenbach, Th. Rummel, K. Risse, Th. Mönnich, H-S. Bosch and the W7-X Team
}

\begin{abstract}
A hypothetical ground fault in the superconducting (sc) magnet system of Wendelstein 7-X would shift the midpoint of the grounding system and therefore increase the voltage to ground during a fast discharge of the sc magnet system. As this voltage could endanger the high voltage integrity of the sc magnet system, an InService-Test system has been developed to monitor the insulation of the sc magnet system during operation especially after changing the loads of the magnets. The system, that is associated to the power supply system, has been designed to lift the electrical potential of non-planar coil system with respect to ground by $1.5 \mathrm{kV}$ and to measure the resulting leak current while the magnets are operated. The leak current is used to evaluate any changes in the insulation resistance and to decide whether a fast discharge can still be done without endangering the sc magnet system. The design of the InService-Test was evaluated with computer simulations of the power supply and the magnet system, and a prototype that was tested at one coil group together with the corresponding power supply. In 2014, the system was finally installed for all five non-planar coil groups and commissioning took place for the first experiment campaign in 2015. Operated during the first experimental campaigns of Wendelstein 7-X from 2015 to 2017 the experiences gained led to modifications that already have been implemented and tested during the operation campaigns in 2018 and 2019.

The paper describes the implementation of the In-Service-Test system and the associated tests for the commissioning as well as the first operational results and corresponding modifications.
\end{abstract}

Index Terms-Insulation, leakage currents, superconducting magnets, high voltage test, Wendelstein 7-X

\section{INTRODUCTION}

$\mathrm{T}$ HE SUPERCONDUCTING MAGNET SYSTEM OF W7-X consists of 50 nonplanar and 20 planar coils. The 70 superconducting magnets are divided into 7 coil groups of ten equal coils connected in series in each group. Every group is powered by an individual power supply and is connected to ground via middle point connection between the feeding bus bars [1]. During energizing and de-energizing operations of the sc magnet system, forces of up to $1.5 \mathrm{MN}$ will lead to deformations of the coil housings in the range of up to $35 \mathrm{~mm}$ thereby stressing the glass fiber reinforced epoxy insulation [2]. During normal operation, even significant damage to the coil

Manuscript received September 2019.

F. Füllenbach, Th. Rummel, K. Riße, Th. Mönnich, and H-S Bosch are with the Max-Planck-Institute for Plasma Physics, 17491 Greifswald, Germany, insulation will not lead to any faulty behavior of the coil, as the leak current has to flow through the conductive coating and/or the coil casing before reaching ground potential. This path will be of a much higher resistance than the superconducting coil group. Therefore, the ground fault could easily stay unnoticed until a high voltage (HV) e.g. during a fast discharge is applied to the coils. To prevent too high voltages during a fast discharge, because of a shifted middle point due to an insulation failure, the In-Service test (IS-test) system has been designed $[3,4]$ and installed into the existing power supply system. Its task is to check the insulation integrity before and after each significant change in the magnet field configuration and to indicate the loss of proper insulation. The IS-test system is built upon five IS-test cabinets dedicated to the five power supply systems for the non-planar coils and a control cabinet. In Fig 1. a principle sketch of the IS-test cabinet integrated into the power supply is shown. During operation, the middle point connection at $\mathrm{K} 1$ to ground will be opened and a HV source is commutated with $\mathrm{K} 2$ into the branch. The HV source will then lift the potential of the whole magnet system with respect to ground and measure the leakage current that is expected with few $\mathrm{mA}$.

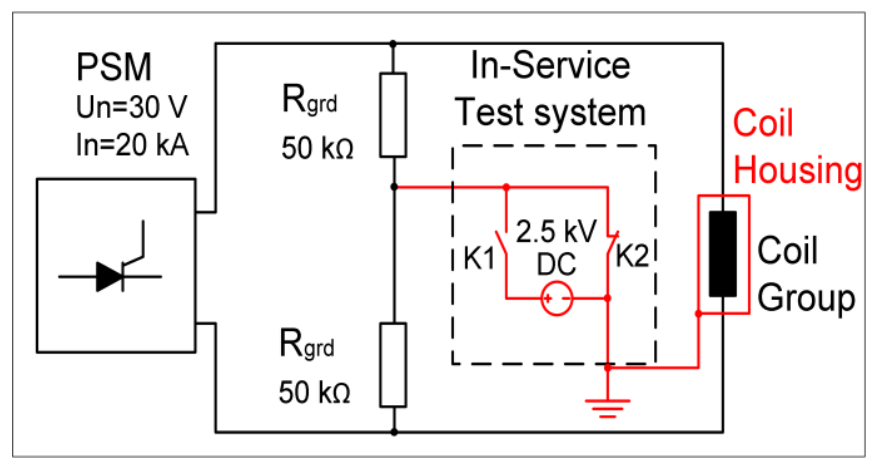

Fig. 1. Principle sketch of the IS test system integrated into the power supply of the superconducting magnets consisting of the power supply module (PSM) and the middle point grounding resistors $\left(\mathrm{R}_{\mathrm{grd}}\right)$

The implementation of the IS test systems, the first experiences during the operation of Wendelstein 7-X different experiment

(corresponding author phone: +49 383488 2776; fax: +49 383488 2709; email: frank.fuellenbach@ipp.mpg.de). 
campaigns (OP1.1, OP1.2a/b) and modifications made will be described in this paper.

\section{INTEGRATION OF THE IS TEST SYSTEM}

For the integration into the power supply system, two interfaces had to be considered: installation of the hardware with the connection to the power supply and the control interlocks with power supply and the overall control system.

A suitable space allocation for the IS-test cabinets beside accessibility was mostly determined by proximity to the ground resistors with middle point connection and the current bus bars. Furthermore the magnetic field levels at the given space allocation had to be taken into account as the power supply is close to the W7-X machine which leads to magnetic field levels of several $\mathrm{mT}$. The switch-gear used for the IS-test system was already tested under magnet fields of up to $10 \mathrm{mT}$ and therefore considered suitable for the task.

For the control, the given safety measures in conjunction with the overall safety control system had to considered. Several parts of the coils services that will be put on HV are not insulated and accessible for personnel. Therefore, a twofold safety strategy is implemented:

- The IS-test systems needs a clearance from the central safety control.

- Additional a local clearance from the power supply is needed that can only be given if the power supply has also a clearance from the central safety control and a certain operation state that is higher than "off". One prerequisite for this operation state is that the torus hall is free of personnel.

\section{COMMISSIONING}

After the installation of the five IS-test cabinets and the IStest control cabinet into the power supply system in 2014, first commissioning test have been performed while $\mathrm{W} 7-\mathrm{X}$ was still under construction. In a first test campaign, all five cabinets have been operated with their corresponding power supplies but without being connected to the magnet system. A ground fault has been simulated by implementing a resistance of $63 \mathrm{k} \Omega$ between the current bus bars and the ground potential. During the process, it became evident, that the leakage current generated by the power supply even without a ground fault present is higher than expected and in a range of $6 \mathrm{~mA}$. Investigations revealed that the IS-test system was integrated in a way that a pair of voltage transducer that are measuring the voltage across the ground resistors are connected to the HV side of the IS-test system. Therefore, they generate a measurement current with their given aspect ratio of 1,000,000:1 leading to $1 \mathrm{~mA} / 1 \mathrm{kV}$ leakage current. A modification in the connecting circuit eliminated this issue, reducing the leakage currents by $5 \mathrm{~mA}$ at $2,5 \mathrm{kV}$. After that, tests were performed without the magnet system and after the completion of W7-X with the magnet system but still at room temperature. The results are shown in Table I.
TABLE I

LEAKAGE CURRENTS DURING COMMISSIONING

\begin{tabular}{ccccc}
\hline \hline $\begin{array}{c}\text { Power } \\
\text { Supply \# }\end{array}$ & $\begin{array}{c}\mathrm{cw} \mathrm{G} \\
{[\mu \mathrm{S} / \mathrm{cm}]}\end{array}$ & $\begin{array}{c}\text { Leakage current } \\
\text { with PS only } \\
{[\mathrm{mA}]}\end{array}$ & $\begin{array}{c}\mathrm{cw} \mathrm{G} \\
{[\mu \mathrm{S} / \mathrm{cm}]}\end{array}$ & $\begin{array}{c}\text { Leakage current } \\
{[\mathrm{mA}]}\end{array}$ \\
\hline AAE10 & 9,03 & 1,55 & 7,49 & 1,65 \\
AAE29 & 7,79 & 1,4 & 7,49 & 1,42 \\
AAE38 & 7,79 & 1,38 & 7,49 & 1,41 \\
AAE47 & 9,03 & 1,41 & 7,46 & 1,42 \\
AAE56 & 9,03 & 1,12 & 7,46 & 1,03 \\
\hline \hline
\end{tabular}

Leakage currents and conductivity of the cooling water (cw) during commissioning with and without magnet system

The values for the leakage current do differ just slightly between the test with (column 3) and without (column 5) the sc magnet system connected. The slight deviations are mostly driven by the different conductivity values for the cooling water, which will range from 1 to $10 \mu \mathrm{S} / \mathrm{cm}$ during operation.

During the commissioning of the power supply system in 2004, HV insulation tests have been performed at $10 \mathrm{kV}$ resulting in leakage currents of about $6 \mathrm{~mA}$. The leakage currents of the sc coil system were measured at several $\mu \mathrm{A}$ per coil group. Therefore, the values in the range of $1.5 \mathrm{~mA}$ for one coil group including its power supply according Table I fit well to the expectation. Nevertheless, this also shows the weakness of the IS test system as the leakage current of the power supply is much higher than the one of the coils. As a result, the minimum ground fault resistance detection threshold of the IS test system needs to be lower than $0.5 \mathrm{M} \Omega$, while the insulation resistance of a single coil to ground is at approximately $1 \mathrm{G} \Omega$.

During the completion of Wendelstein 7-X in 2015 the overall control system of the IS-test system was established and was ready for operation with the beginning of the commissioning of the whole magnet system. In Fig 2 the overall control system of the power supply is shown with the added programmable logic controller (PLC) for the IS-test system. The connections to the data acquisition and to the segment control, have not been realized in the first operational phase of W7-X but only later on in OP1.2.

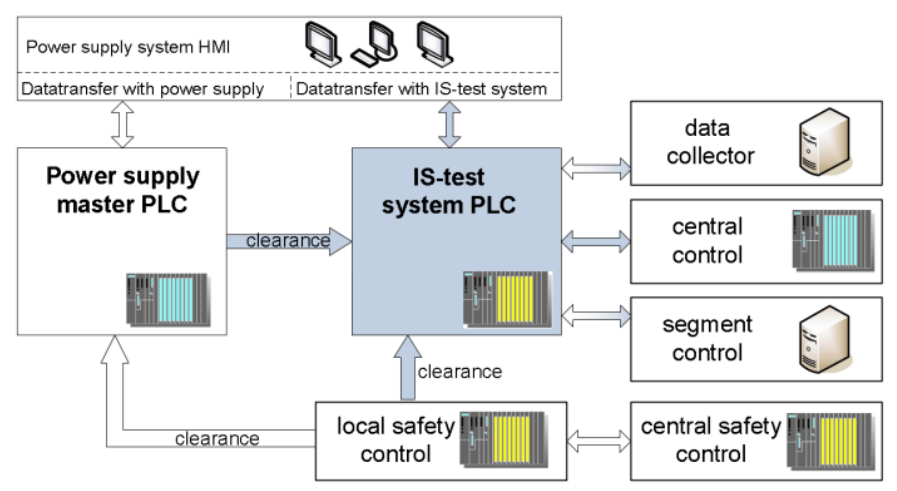

Fig. 2. Overall control system of the power supply. The added components and connections are shown in blue

In order not to stress the magnet system more than necessary it was decided to set certain voltage levels for the IS -test depending on the expected voltage levels during a fast discharge of the magnet system. For easier handling in the control system, the corresponding operating coil current is 
being used. For the commissioning, this was set to $500 \mathrm{~V}$ for every $4 \mathrm{kA}$. As the maximum current used during OP 1.1 was $12.8 \mathrm{kA}$ the maximum IS-test voltage was set to $2000 \mathrm{~V}$. During commissioning levels of 500, 1000, 1500 and $2000 \mathrm{~V}$ have been used. Fig. 3 shows the average leakage currents for each power supply at the given voltage levels. It can be seen that, as expected the leakage currents behave mostly linear and without any additional discharge effects at higher voltages. The deviation from the linear function comes mainly from the changes of the cooling water conductivity that has been lower during the day the tests with $1500 \mathrm{~V}$ have been made..

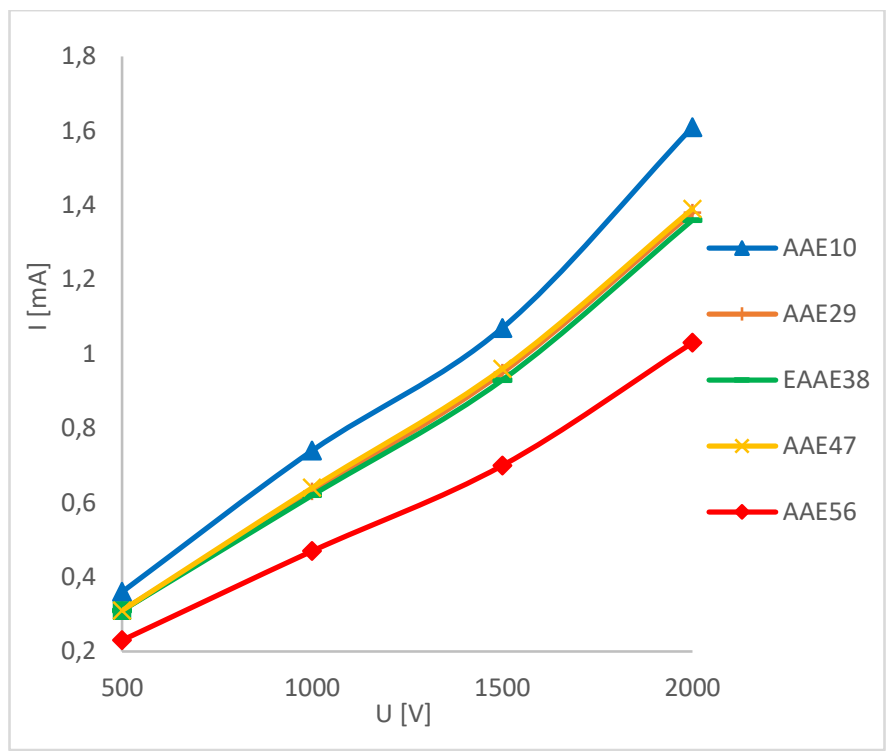

Fig. 3. Leakage current with respect to the test voltage used during commissioning of the magnet system

\section{MODIFICATION}

During OP1.1 the leakage currents measured during the ramp up of the HV have not been considered for the evaluation of the insulation. The capacitive part of the current during that phase is too high to put it under the same restriction as the flattop current. Nevertheless, it has to be taken into account as the leakage current due to fading insulation will already exist at this point. Therefore, it was decided to add a monitoring also during the ramp up. During this sequence certain max. current has to be set to ensure that the IS-Test can be operated safe and without endangering a coil that already has a ground fault present. For the flat top on the other hand, the evaluation has been changed from leakage current to insulation resistance, adding the respective calculation to the plc software of the IStest system. This will make the evaluation easier because it is independend from the used test voltage. Furthermore, the threshold values for the insulation evaluation have been refined. During ramp up and ramp down a fixed current value based on measurements has been set. For the insulation during the flat top a value of $1 \mathrm{M} \Omega$ has become the limit for considering the insulation as sufficient for further operation.

The test sequence starts a self test whereas the HV source drives the full current of $40 \mathrm{~mA}$ at a $63 \mathrm{k} \Omega$ resistance. After the commutation into the ground path, the voltage is set to $1200 \mathrm{~V}$ within $2 \mathrm{~s}$. The current reaches a peak of about $1.5 \mathrm{~mA}$ and settles at $0.84 \mathrm{~mA}$ after less than $1 \mathrm{~s}$. This current then is taken to determine the insulation resistance to be compared with the threshold value. In Fig. 4 the test sequence is shown without the self test.

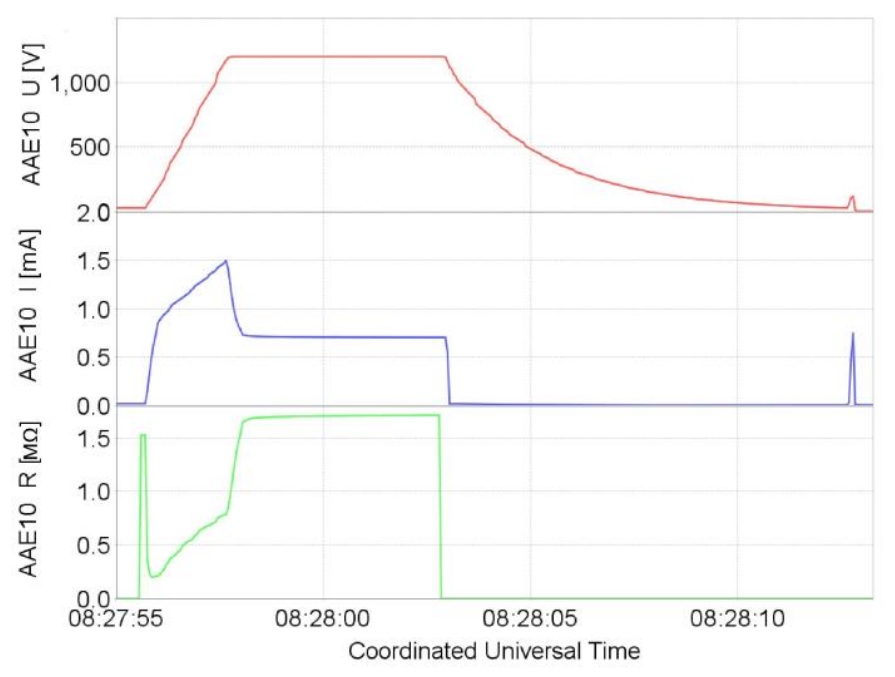

Fig. 4 Voltage (red), current (blue) and insulation resistance (green in $M \Omega$ ) during a test sequence of coil group 1 with power supply AAE10 in OP 1.2

After OP 1.2a the connection to the data collector (see Fig. 2) has been established and successfully tested during OP $1.2 \mathrm{~b}$ in 2018. From that point on the IS-test system was contemplated to be finalized.

\section{EXPERIENCES DURING OPERATION}

Since the commissioning of the IS-test system, it has been used throughout the three experiment campaigns OP1.1, $\mathrm{OP} 1.2 \mathrm{a} / \mathrm{b}$ of $\mathrm{W} 7-\mathrm{X}$. The superconducting magnet system is not already integrated into the automated segment control for the W7-X, and therefore operated manually. The same is valid for the IS-test system. For the operation the following parameters have been determined to decide whether a current change is significant to trigger an IS-test:

- The current change has to be at least $1 \mathrm{kA}$.

- When having several successive small changes in the sum of the absolute values has to be at least $1 \mathrm{kA}$.

- When having different changes in several coil groups the one with the highest sum of the group determines the necessity of an IS-test (IS-test will always be done for all five non planar coil groups).

The leakage current threshold value for considering a ground fault in the sc magnet system was initially calculated by the known leakage currents adding a leakage current based on the cooling water conductivity and multiplying the result with two.

\section{A. $O P 1.1$}

With the parameters used during the commissioning, the IStest system was operated from the beginning of OP1.1. Fig. 5 shows the results of all IS-test shots during the first campaign. For all five coil groups the leakage current stayed constant at their respective levels with very few exceptions which are single events. These can be explained by the fact that the measured data was recorded manually during OP1.1 and that 
the IS-test systems always gives min. and max. values during the HV flat top to identify the capacitive overshoot current at the end of the ramp up. It is assumed that in the cases where the values do differ, the maximum value has been accidently recorded. Nevertheless, the threshold for identifying a critical loss of insulation was set to two times the average values during normal operation and are not exceeded at any time.

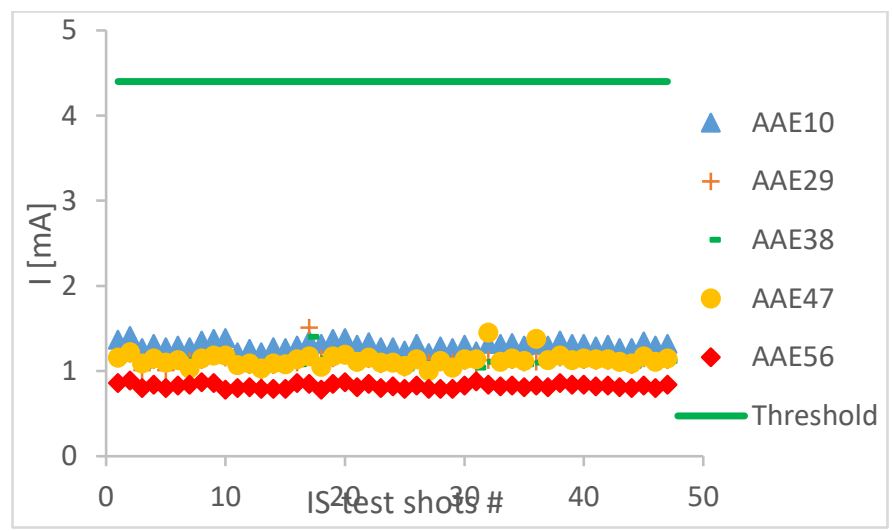

Fig. 5. Leakage currents during OP1.1 for all five nonplanar coil systems

\section{B. $O P 1.2$}

For OP1.2 the voltage levels for the IS-test have been modified according to the expected max. voltage during a fast discharge that was reduced due to a modification of the protection system for the sc magnet system [5]. Up to $10 \mathrm{kA}$ the IS- test voltage is set to $500 \mathrm{~V}$, from $10 \mathrm{kA}$ up to $13 \mathrm{kA}$ to $1000 \mathrm{~V}$ and above $13 \mathrm{kV}$ to $1200 \mathrm{~V}$. Furthermore, the threshold values for considering a ground fault have been adjusted as well and are now fix for the leakage current during ramp up at $2 \mathrm{~mA}$ and for the insulation resistance for all coil groups at $1 \mathrm{M} \Omega$ for the flat top. In addition, the parameter for triggering the IS-test was raised from $1 \mathrm{kA}$ to $2 \mathrm{kA}$. In Fig. 6 an extract of the measured insulation resistances during of OP1.2 are shown.

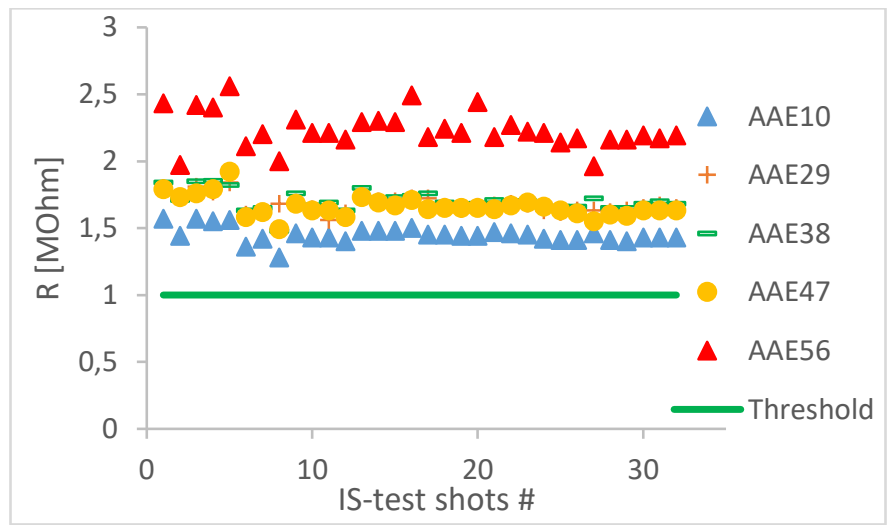

Fig 6. Insulation resistance during OP1.2 for all five nonplanar coil systems

Only the coil group connected to the power supply AAE56 shows higher variations for the insulation resistance in a range of $\pm 10 \%$. This can be explained by the exposed location of this particular power supply that supports ambient influences like temperature, humidity and air pressure. It can be assumed that the insulation for the coils themselves, remains firm for all five coil groups.

\section{SUMMARY}

After the design, proof of principle and prototyping, the IStest system has been installed into the power supply facility of Wendelstein 7-X and has been connected to the sc magnet system as well as to the control system. It was successful operated during the first three experiment campaigns.

Several modifications have been established to improve the ground fault detection, the safety of the magnet system and data acquisition. Within the given limitation, the IS-test system has proven to be a valuable tool to monitor the insulation of the magnet system with respect to significant ground faults during the operation of the magnets.

Further investigations to determine the sources of the leakage currents in the power supply system increasing the sensitivity of the measurements are planned prior to OP2 of Wendelstein $7-\mathrm{X}$ in 2021.

\section{ACKNOWLEDGMENT}

This work has been carried out within the framework of the EUROfusion Consortium and has received funding from the Euratom research and training programme 2014-2018 and 2019-2020 under grant agreement No 633053. The views and opinions expressed herein do not necessarily reflect those of the European Commission.

\section{REFERENCES}

[1] Th. Rummel, et al, "Power supplies for the Wendelstein 7-X stellarator", Fusion Engineering and Design, 66-68 (2003), pp. 1115-1118

[2] V. Bykov, et al., Structural analysis of W7-X: Overview, Fusion Eng. Des. 84, 215-219 (2009)

[3] Füllenbach, F.; Rummel, T.; Mönnich, T.; Feasibility Study of a High Voltage In-Service-Tests on the Superconducting Magnet System of Wendelstein 7-X. IEEE Transactions on Applied Superconductivity 22 (3), (2012)

[4] Füllenbach, F.; Rummel, T.; Mönnich, Design and Prototype of the high voltage In-Service-Tests on the superconducting magnet system of Wendelstein 7-X

[5] K. Risse et al., „Updates on protection system for Wendelstein 7-X superconducting magnets," Fusion Engineering and Design, vol. 146, pp.910-913, 2019.Doi: 10.1016/j.fusengdes.2019.01.111 